\title{
DEL PROYECTO ERA A LAS EXPERIENCIAS DE LAS COMUNIDADES AUTÓNOMAS: AVANCES EN EL DESARROLLO DE SISTEMAS DE RECONOCIMIENTO, EVALUACIÓN Y ACREDITACIÓN DE COMPETENCIAS*
}

\author{
SINCE ERA PROJECT TO AUTONOMIC COMMUNITIES: ADVANCE \\ IN THE THEVELOPMENT OF COMPETENCES ACCREDITATION, \\ EVALUATION AND RECOGNITION SYSTEMS
}

\author{
Cristina Miranda Santana** y Arcadia Martín Pérez***
}

Universidad de Las Palmas de Gran Canaria

\section{RESUMEN}

El desarrollo de los sistemas de reconocimiento, evaluación y acreditación de competencias en España no tiene carácter oficial, aún así se han realizado algunas experiencias. Este artículo presenta las diferentes tentativas que hasta el momento se han realizado en España, analiza cómo las experiencias implementadas por las Comunidades Autónomas mantienen las líneas desarrolladas en ERA (Proyecto de evaluación, reconocimiento y acreditación) y aporta algunas conclusiones que pueden orientar la conceptualización y el desarrollo práctico de estos sistemas.

Palabras clave: Acreditación, Sistemas de reconocimiento, competencias profesionales, evaluación de competencias, métodos de acreditación, aprendizaje a lo largo de la vida, formación profesional, vocational guidance.

\section{ABSTRACT}

The development of evaluation, recognition and accreditation of competences system in Spain hasn't got any official recognition, despite having had some work done in this area. This article shows the

\footnotetext{
* Este trabajo es fruto del desarrollo de un proyecto precompetitivo financiado por la Universidad de Las Palmas de gran Canaria, denominado Sistemas de Acreditación. La entrega del informe final se realizó en 2008. Grupo de Investigación en Educación Social (GIES).

** Profesora Titular de Universidad. Universidad de Las Palmas de Gran Canaria. Dra. en Psicopedagogía. Líneas de investigación: Orientación y formación profesionalizadota E-mail: cmiranda@dedu.ulpgc.es.

*** Profesora Contratada Doctora. Universidad de Las Palmas de Gran Canaria. Dra. en Psicopedagogía. Líneas de Investigación: Orientación y formación profesionalizadota E-mail: amartin@dedu.ulpgc.es.
} 
different experiences achieved up to now in Spain, analyzing how the works developed in the Autonomic Communities follow the guidelines developed in the accreditation, recognition and evaluation project (ERA), providing some conclusions which may serve as a guidance in the conceptualisation and practice in the development of this system.

Key Words: Accreditation, evaluation and recognition systems, professional competences, evaluation competences, methods of accreditation, long-life training, vocational training, vocational guidance.

\section{Introducción}

En nuestro contexto, la Ley Orgánica 5/2000, de 19 de junio de las Cualificaciones y de la Formación Profesional crea el Sistema Nacional de Cualificaciones y Formación Profesional y define un marco normativo que posibilita realizar la evaluación, reconocimiento y acreditación oficial de las cualificaciones profesionales cualquiera que hubiera sido la forma de obtenerlas.

Este marco legal propicia la elaboración y desarrollo del Proyecto de evaluación, reconocimiento y acreditación (Proyecto ERA, 2003). Se trata de un proyecto experimental promovido por el Ministerio de Educación y Ciencia, cofinanciado por el Fondo Social Europeo, dirigido desde la Subdirección General de Formación Profesional y con la participación de siete Comunidades Autónomas (Andalucía, Castilla-La Mancha, Galicia, Murcia, Navarra, País Vasco y Valencia), del Ministerio de Trabajo y Asuntos sociales, a través del Instituto Nacional de Empleo (INEM) y de los Agentes Sociales. El valor de esta experiencia reside, desde el punto de vista de las políticas educativas y laborales, en derivar de ella una norma general que sirva de marco para el desarrollo del Sistema de Reconocimiento, Evaluación y Acreditación de la Competencia en todo el territorio estatal.

La experiencia adquirida a través del Proyecto ERA (2003) juntamente con la disposición transitoria única de Evaluación de las competencias profesionales lleva, en principio, a algunas comunidades autónomas como la Vasca o la Catalana a promover desde el año 2004 experiencias de acreditación. En la actualidad ambas comunidades han sido capaces de consolidar un Sistema de Reconocimiento, Evaluación y Acreditación de la Competencia que aún compartiendo elementos fundamentales presentan características propias. Las Comunidades Autónomas de Aragón (2006), Castilla-La Mancha (2007) y Canarias (2007) también se han incorporado a este tipo de experiencias aunque su corta trayectoria, reducida a una sola convocatoria, ha dado lugar a que las instituciones que tienen responsabilidad en el desarrollo de los sistemas y procesos de acreditación se inicien en la puesta a punto y establecimiento de condiciones que exigen un proceso tan complejo como éste, pero sin duda alguna cargado de un gran valor social.

Este trabajo intenta dar a conocer el desarrollo que los Sistemas de reconocimiento, acreditación y evaluación de la competencia han tenido en España hasta ahora.

Con este objeto analizamos en qué medida los modelos o propuestas de reconocimiento, evaluación y acreditación de competencias desarrollados por cada una de las comunidades autónomas mantienen o mejoran las conclusiones alcanzadas en el Proyecto ERA (2003). Este análisis lo respaldamos, tanto en la información extraída de las normas que han regulado las diferentes experiencias como en los informes oficiales que al respecto se han emitido. Además, estos datos han sido contrastados y complementados a través de entrevistas realizadas a informantes claves en cada una de estas Comunidades Autónomas. 


\section{Análisis de las experiencias desarrolladas en las Comunidades Autónomas respecto a ERA}

El estado español carece de norma que desarrolle a nivel estatal el sistema de reconocimiento, evaluación y acreditación de competencias profesionales. Esta norma se constituirá como un referente al que las CCAA deberán remitirse para desarrollar experiencias de acreditación en sus respectivos territorios. La realidad es que muchas de las comunidades autónomas ven como necesidad experimentar y analizar sistemas de reconocimiento, evaluación y acreditación de la competencia con el objeto de calibrar las posibilidades y las necesidades estructurales de cada una de ellas en el proceso de generalización del sistema.

En esta ocasión centramos el análisis en los componentes básicos que dan forma a las diferentes fases del proceso de acreditación de la competencia y decidimos, por motivos de extensión del documento, no abordar el estudio de los elementos fundamentales que configuran el Sistema de Acreditación.

La matriz análisis comparativo del procedimiento de evaluación, reconocimiento y acreditación en las CCAAs, que presentamos a continuación, describe de manera sintética los componentes básicos que configuran las fases del proceso de acreditación. Asimismo, cabe indicar que hemos dado a las fases un rótulo general que en esencia responde a las denominaciones dadas por cada CCAA aunque con pequeñas variaciones que afectan más a su designación que a su significado (fase de información, orientación, evaluación y acreditación).

La matriz, como se puede observar, permite contrastar el procedimiento de evaluación, reconocimiento y acreditación seguido en cada una de las experiencias. Un primer análisis nos lleva a afirmar que el procedimiento seguido en las diferentes experiencias guarda entre ellas una gran similitud. Sin embargo, el estudio detenido de cada una nos indica que no siempre es así apreciándose algunos matices que pasamos a comentar.

En la fase de información comprobamos que en relación al objetivo previsto las experiencias de tipo experimental (Proyecto ERA, Castilla-La Mancha, Aragón y Canarias) no sólo informan del sistema de acreditación de la competencia sino que además insisten en dar a conocer el proyecto del que se deriva la experiencia y el Sistema de Cualificaciones y Formación Profesional. De esta manera, el tipo de contenido que tratan en cada una de ellas se concentran, en unos casos, única y exclusivamente en el proceso de reconocimiento, evaluación y acreditación de competencias mientras que en otros (País Vasco y Catalán), además, tratan aspectos que van más allá de este proceso y se centran en el Sistema de Acreditación.

En esencia entre el Proyecto ERA y el resto de las experiencias realizadas hasta ahora se observa un elemento diferencial. La información en el Proyecto ERA queda dentro del mismo.

Proyecto, mientras que en el resto parece que se ha externalizado. Este hecho nos lleva a pensar y a suponer que la fase de información aún siendo un elemento esencial para el éxito de las experiencias no forma parte del proceso de reconocimiento, evaluación y acreditación de competencias profesionales.

De manera indirecta, esta situación también tiene consecuencias en el profesional al que se le atribuye la responsabilidad de informar pues tan sólo el Proyecto ERA y Aragón, atribuyen la labor informadora a un profesional con cualificación específica en ésta. En el resto de las situaciones, la tarea es asumida por administrativos (expertos en procesos administrativos) que en el mejor de los casos han recibido una formación específica. 


\begin{tabular}{|c|c|c|c|c|}
\hline 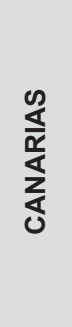 & 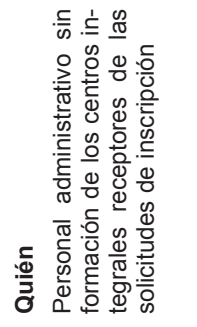 & 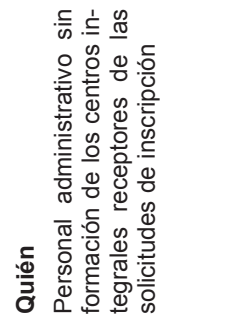 & 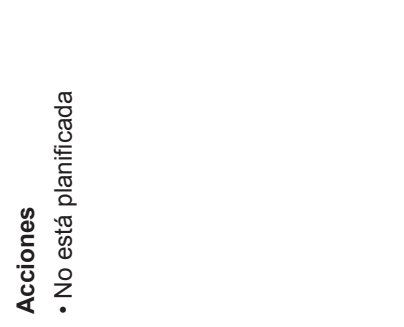 & 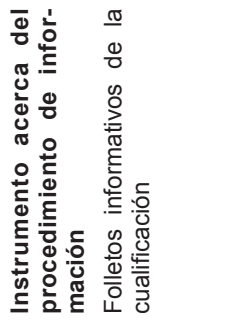 \\
\hline 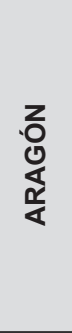 & 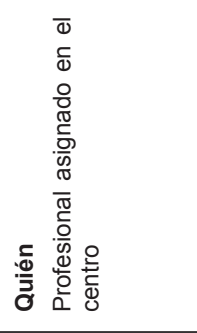 & 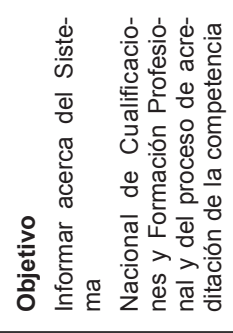 & 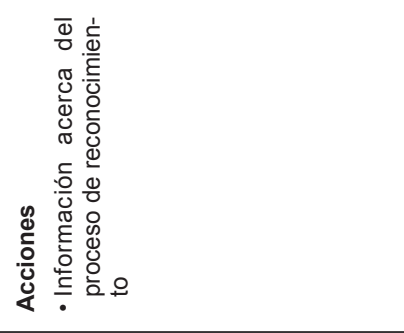 & 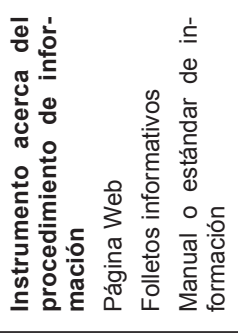 \\
\hline 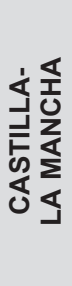 & 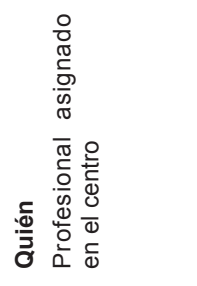 & 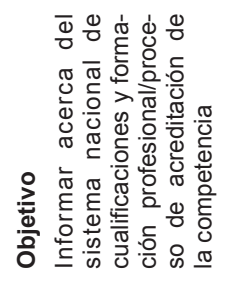 & 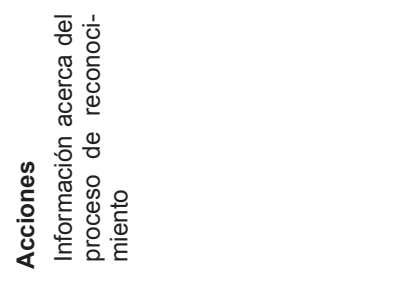 & 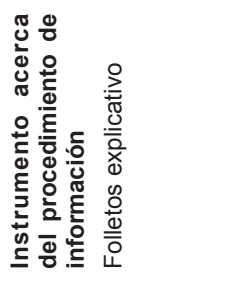 \\
\hline$\frac{\mathbb{s}}{3}$ & 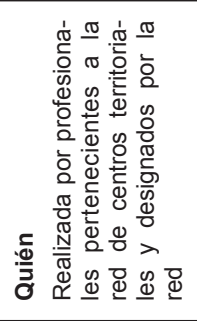 & 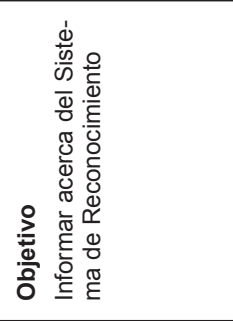 & 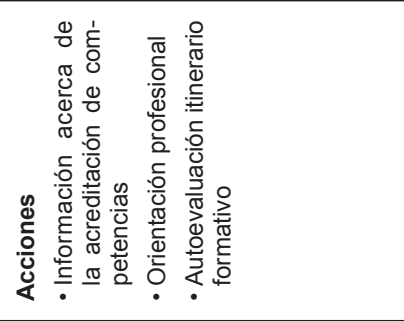 & 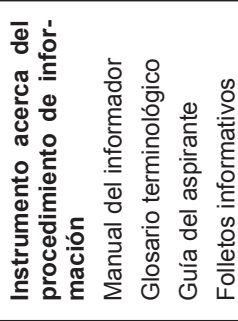 \\
\hline 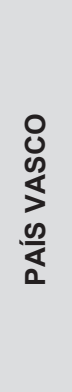 & 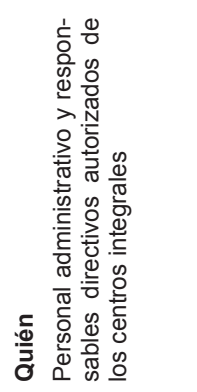 & 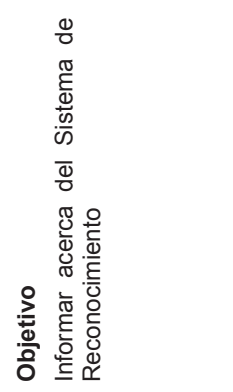 & 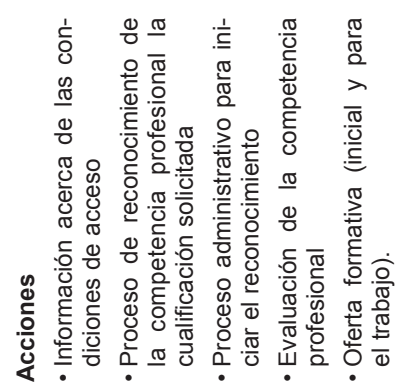 & 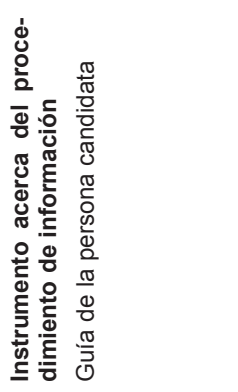 \\
\hline
\end{tabular}




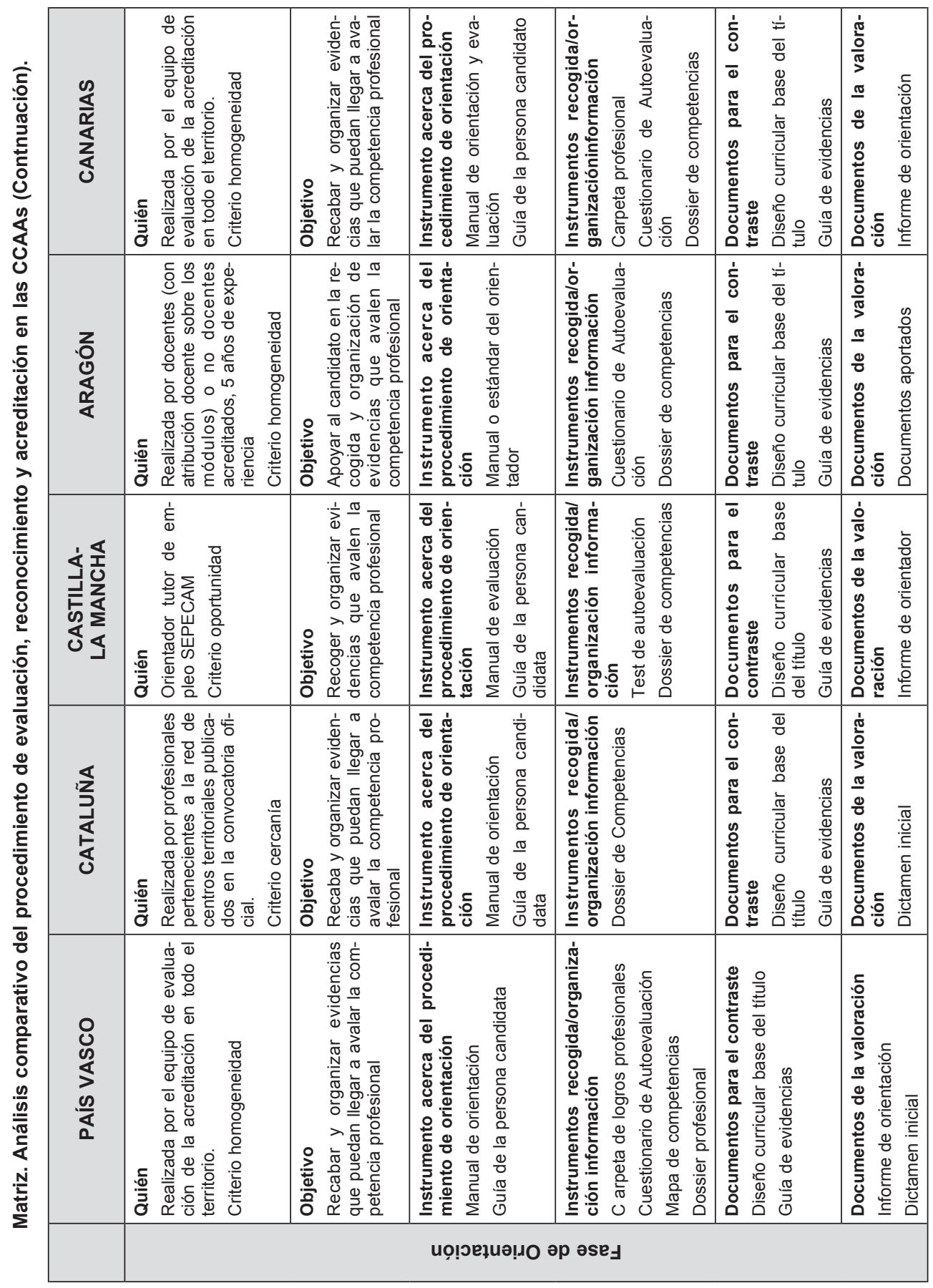




\begin{tabular}{|c|c|c|c|c|}
\hline 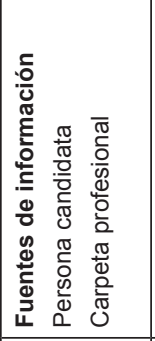 & 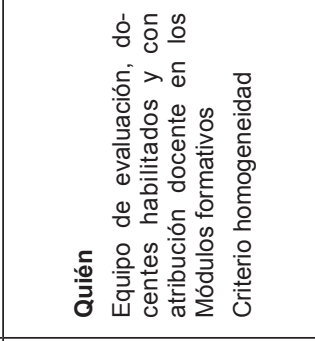 & 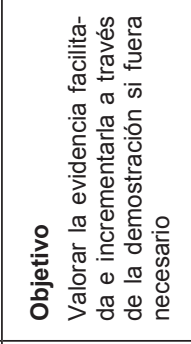 & 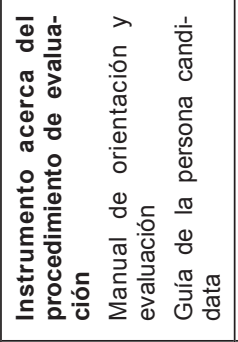 & 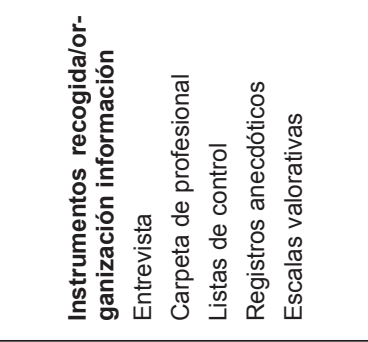 \\
\hline 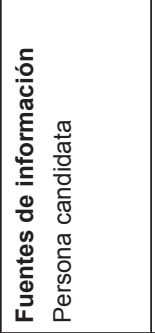 & 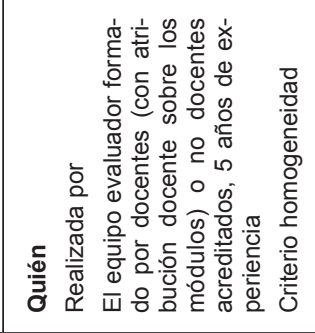 & 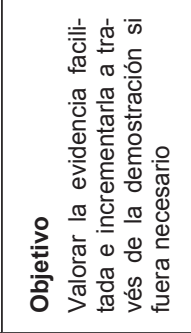 & 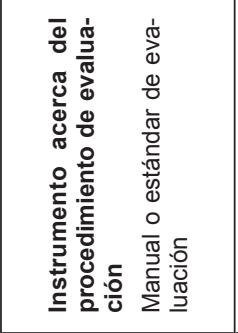 & 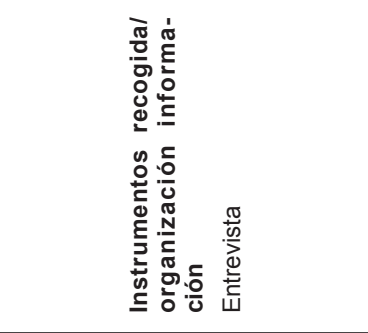 \\
\hline 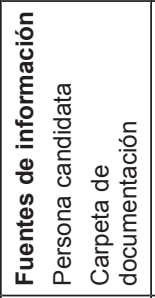 & 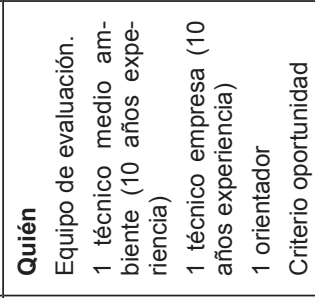 & 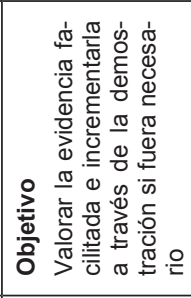 & 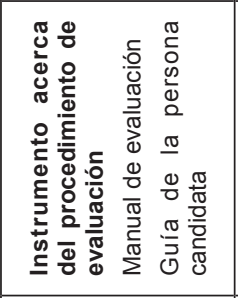 & 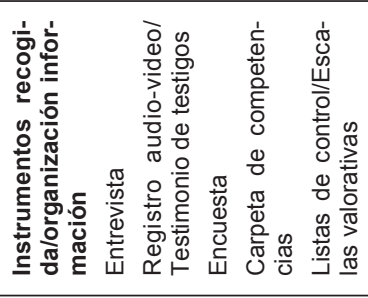 \\
\hline 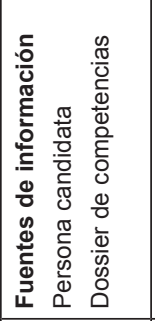 & 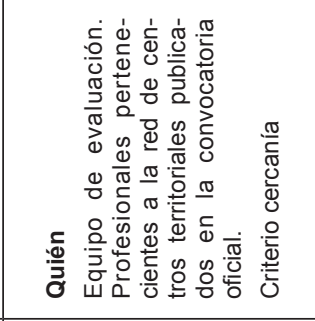 & 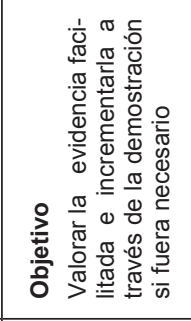 & 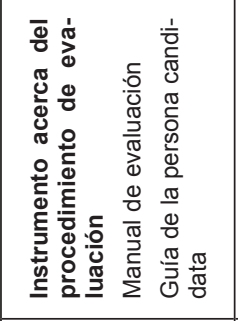 & 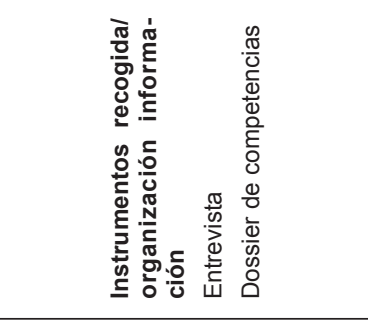 \\
\hline 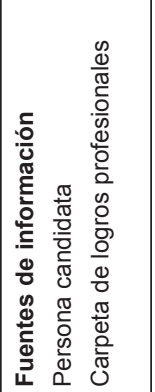 & 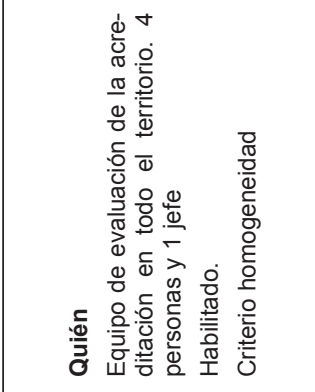 & 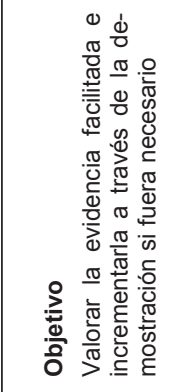 & 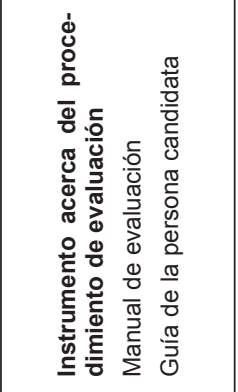 & 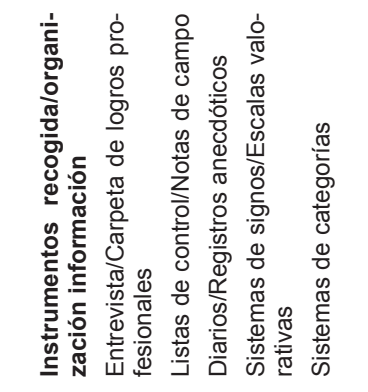 \\
\hline
\end{tabular}




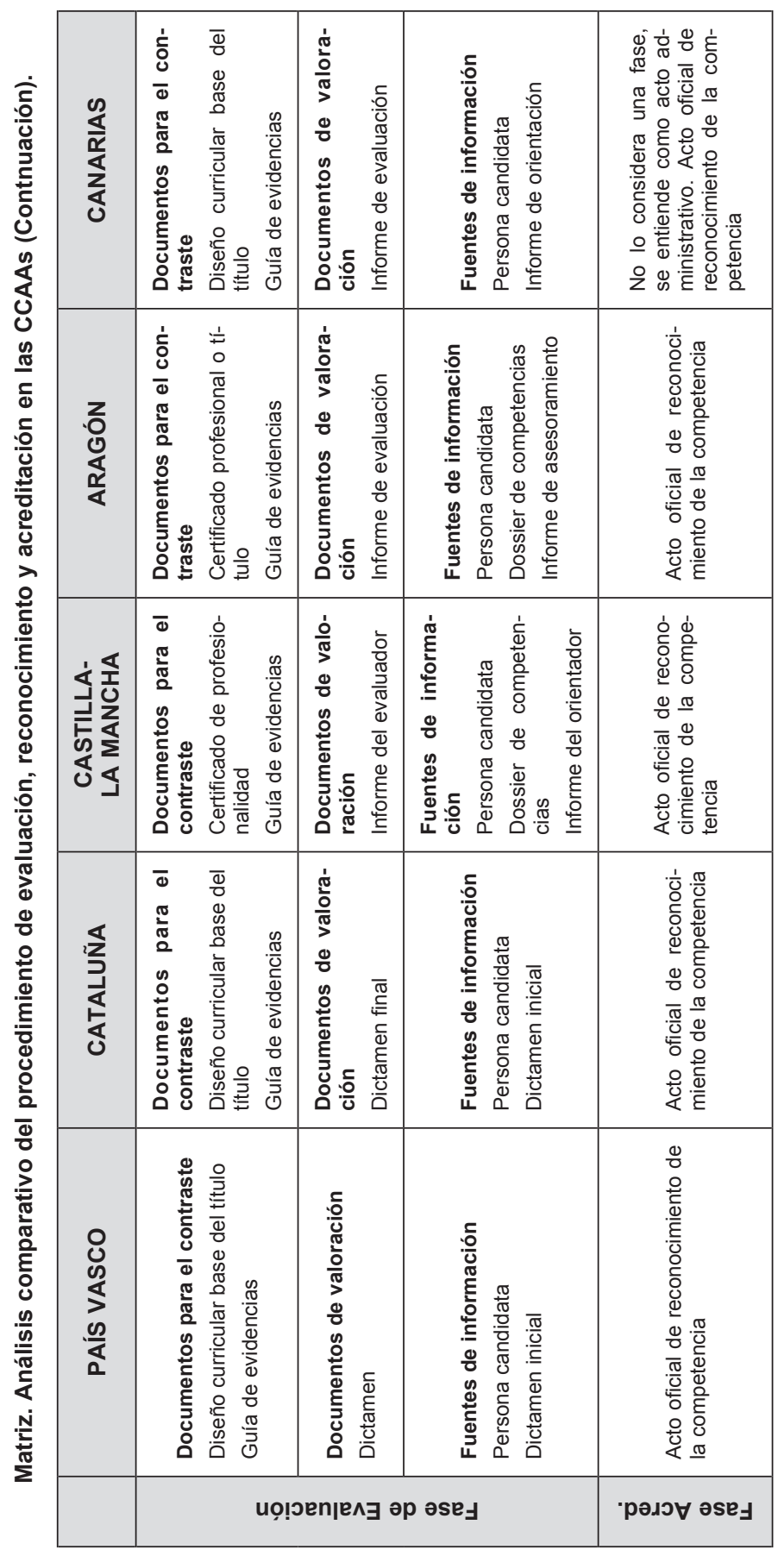


Respecto a la fase de orientación comprobamos que entre el Proyecto ERA y el resto de las experiencias desarrolladas en las CCAAs no hay diferencias en la concepción del objetivo que se proponen, ni en los instrumentos utilizado en el procedimiento de orientación, ni en los documentos aplicados en el contraste y tampoco existen diferencias en las fuentes de información. En cuanto al último aspecto comentado, debemos saber que en esencia, cuando hablamos de carpeta de logros, de documentación o profesional hacemos referencia a lo mismo y que estas carpetas están compuestas entre otros documentos por el dossier de competencias en el sentido previsto por diferentes autores (Ibarra y Sánchez Rojas, 1999; Arraiz y Sabirón, 2007). Ahora bien, observamos como aportación al Proyecto ERA el que todas las comunidades autónomas hayan desarrollado un manual (manual de orientación, estándar de evaluación e incluso manual de evaluación) que permita al profesional que asuma esta fase transitar por ella con acierto y, también, con cierta seguridad.

Diferencias sustanciales de análisis las encontramos en los profesionales, en los instrumentos utilizados en la recogida/organización de información y en los documentos de valoración. En los profesionales que realizan la orientación reside la mayor disparidad entre experiencias. Tanto el Proyecto ERA como la experiencia desarrollada en Castilla-La Mancha consideran que los profesionales que deben llevar a cabo el proceso de orientación son expertos en orientación. Ahora bien, es necesario indicar que el objetivo propuesto en ERA para esta fase "analizar la trayectoria formativa de la persona candidata y prepararla para el proceso ERA" parece responder a la cualificación de este profesional; sin embargo, el objetivo previsto en Castilla-La Mancha "recoger y organizar evidencias que avalen la competencia profesional" podríamos decir que se aleja del saber hacer de estos profesionales. El resto de las experiencias optan por profesionales expertos (docentes o en ejercicio) en la cualificación profesional y en su defecto en la familia profesional objeto de acreditación ajustándose perfectamente al objetivo previsto.

Todas las experiencias incluso ERA coinciden en proponer el test o cuestionario de autoevaluación y el dossier de competencias como instrumentos utilizados en la recogida/organización de información a excepción de Cataluña que opta exclusivamente por el Dossier de competencias. El País Vasco, además, dispone del mapa conceptual y éste y Canarias incorporan la Carpeta de logros profesionales o profesional.

Por último, comentamos los documentos de valoración. ERA, País Vasco, Castilla-La Mancha, Aragón y Canarias optan por el informe como documento que recoge la valoración de la persona candidata. El País Vasco y Cataluña, además, hablan de dictamen inicial. Ahora bien, la realidad es que todos realizan dictamen que queda incluido en el primer informe que se realiza en el proceso a excepción de Castilla-La Mancha.

Esta última relación juntamente con el tipo de profesional que se responsabiliza de esta fase, nos hace pensar acerca de la evolución que ha tenido esta fase en el proceso de evaluación de la competencia. Hablar de un dictamen inicial supone realizar una primera valoración o evaluación derivada de las evidencias indirectas o directas recogidas en esta fase. Esto nos confirma en la idea de que tiene que ser un profesional experto quien se ocupe de esta tarea pues sólo puede dictaminar quien es competente en un campo profesional.

En relación a cuándo se emite este dictamen hemos visto un planteamiento diferente en las experiencias autonómicas respecto al Proyecto ERA. En todas ellas, este primer informe se emite al final de la fase de orientación mientras que en ERA se aborda una vez anali- 
zada la trayectoria formativa de la persona candidata y antes de la elaboración del dossier de competencias. Este cambio supone un avance en la medida en que incrementa la validez del proceso y mejora la percepción que se tiene acerca del criterio o juicio emitido por el orientador.

La fase de evaluación, al igual que en la fase anterior, en ésta existe entre las diferentes experiencias una coincidencia plena con el objetivo y los documentos de contraste. También existe coincidencia con las fuentes de información, con los instrumentos utilizados en el procedimiento de evaluación y con los documentos de valoración pero es necesario realizar algunas matizaciones.

Todas las experiencias identifican como fuente de información a la persona candidata. Ahora bien, la documentación consultada acerca de las diferentes experiencias nos indica que efectivamente la persona candidata es la fuente directa de información pero que además, el equipo o evaluador en el proceso de evaluación se apoya en las evidencias indirectas, que quedan recogidas en las carpetas profesionales, o en su ausencia en los dossier de competencias, y también en los informes de orientación.

Cada experiencia da a los documentos de valoración diferentes denominaciones aunque todos los evaluadores informan y su informe finaliza con un dictamen de evaluación. Por último, clarificamos qué ocurre con los instrumentos de recogida y organización de la información. La realidad es que se emplean los mismos instrumentos que se han utilizado en la fase de orientación (entrevistas y carpetas) más otros que responden a las exigencias de las pruebas de evaluación.

Se confirma la propuesta realizada en el Proyecto ERA acerca de los profesionales que realizan la evaluación pues coinciden en que los evaluadores deben ser profesionales ya sean docentes o en ejercicio con una experiencia profesional mínima en la cualificación y en su defecto en la familia profesional. La aportación al Proyecto ERA, en esta ocasión, reside en la propuesta de un equipo de evaluación, en el que siempre existe la figura de un jefe o coordinador del equipo más otros evaluadores. La cantidad de evaluadores habitualmente está en función de la cantidad de unidades de competencia que la persona candidata se dispone a acreditar y de la atribución docente de las mismas.

Castilla-La Mancha es la única comunidad autónoma que incorpora al equipo de evaluación un orientador, que realiza funciones de secretaría, levantando acta de las decisiones tomadas respecto a cada persona candidata.

La fase de acreditación, es vista por las diferentes experiencias, excepto por Canarias, como la última fase del proceso de reconocimiento, evaluación y acreditación y entendida como un acto oficial en el que se deja constancia del dominio de la unidad de competencia.

Canarias considera que la certificación de la competencia forma parte del proceso de evaluación, mientras que la acreditación de la competencia queda fuera de éste por ser un acto administrativo que pasa a formar parte del Sistema de reconocimiento, evaluación y acreditación de la competencia.

\section{Conclusiones}

Tras el análisis realizado y a modo de conclusión podemos afirmar, cuatro años después y con 5 experiencias en diferentes comunidades autónomas, dos de ellas con más de 10 
convocatorias, que el Proyecto ERA aunque no ha conseguido transferir a las políticas normalizadas los procesos de reconocimiento, evaluación y acreditación de la competencia en el territorio estatal si ha hecho posible generar, en las Comunidades Autónomas que han participado, el interés social y económico que tiene acreditar la competencia a través de vías diferentes a la educación formal, el saber hacer necesario que permita su aplicación, así como la solidaridad necesaria entre comunidades para transferir los hallazgos alcanzados y hacer posible su adaptación a cada contexto autonómico.

La realidad es que el diseño metodológico realizado en ERA para la evaluación de la competencia profesional, en esencia ha sido adoptado por las diferentes Comunidades Autónomas. Además, mantienen los principios básicos que sustentan el procedimiento de reconocimiento, evaluación y acreditación de la competencia previstos en ERA.

Por último y a partir del estudio y contraste realizado en la elaboración de este artículo, nos gustaría realizar algunas aportaciones de interés que hagan posible avanzar en el desarrollo de los sistemas y procesos de acreditación de la competencia profesional:

1. Es necesario distinguir entre el proceso de evaluación de la competencia profesional y el Sistema de reconocimiento, evaluación y acreditación. Cuando hacemos referencia al Sistema hablamos de un proceso de evaluación de la competencia profesional junto a todos aquellos elementos que hacen posible dicho proceso y además, a la estructura organizativa, los centros y profesionales responsables de su desarrollo. De hecho, las Comunidades Autónomas de Cataluña y País Vasco se caracterizan no sólo, por tener consolidados los procesos de reconocimiento, evaluación y acreditación de la competencia profesional, sino también por tener un amplio desarrollo del sistema de reconocimiento, evaluación y acreditación de la competencia profesional.

2. Otro de los aspectos en los que debemos insistir es en hacer visible y clarificar el tipo de actividad que se realiza para llevar a cabo el reconocimiento, evaluación y acreditación. Nuestra propuesta insiste en que la actividad desarrollada es, en su conjunto, de carácter evaluativo en la que su objeto de evaluación son las competencias profesionales. Este motivo nos lleva a hablar, por tanto, de evaluación de la competencia profesional y no de reconocimiento, evaluación y acreditación.

Entendemos que este proceso de evaluación de competencias tiene como finalidad certificar competencias profesionales y garantizar el éxito de dicha evaluación, por lo que requiere generar previamente una evaluación de orientación para valorar las posibilidades de la persona candidata en la evaluación de certificación. Estamos, por tanto, identificando dos evaluaciones distintas pero que existe entre ellas una relación de facilitación. La primera, evaluación de orientación, que coincidiría con lo que se viene denominando fase de orientación, tendría como finalidad evaluar a la persona candidata sobre sus posibilidades de éxito en el proceso de certificación posterior en función de la distancia existente entre el perfil profesional y las evidencias aportadas por la persona candidata. La segunda es una evaluación de certificación, lo que se denomina como fase de evaluación, cuya finalidad es certificar las competencias profesionales solicitadas por la persona candidata a partir de las evidencias aportadas.

3. En consecuencia con lo dicho en el apartado anterior, optamos por reconocer la acreditación como un acto de tipo administrativo que forma parte del sistema de 
acreditación y no del proceso de evaluación contrariamente a como se recogen en las diferentes experiencias autonómicas y en el Proyecto ERA.

4. La difusión y la información específica del proceso de evaluación de la competencia exige el desarrollo y consolidación de un elemento esencial en el Sistema de Cualificaciones y Formación Profesional como es el sistema de orientación profesional. Los institutos de cualificaciones profesionales juegan un papel esencial en todo ello.

5. Los profesionales responsables de la información deben ser trabajadores con cualificación específica, vinculados a los sistemas de orientación y formados en este ámbito.

6. Los profesionales que llevan a cabo la orientación y los que asumen la evaluación deben ser profesionales expertos en la cualificación y en su defecto en la familia profesional. Además, deben estar formados específicamente en evaluación de competencias profesionales.

7. El cuestionario de autoevaluación, el dossier de competencias junto a la carpeta parecen ser instrumentos idóneos en la recogida de información destinada a la evaluación de la competencia y en desencadenar entre el evaluador y persona candidata los procesos de reflexión necesarios para ajustar las expectativas de reconocimiento de la persona candidata en función de su trayectoria sociolaboral.

8. En determinados casos y siendo conscientes de la complejidad que este tipo de evaluación conlleva, la observación en el puesto de trabajo es una situación de enorme valor para inferir el dominio de competencias. Por tanto, es necesario generar instrumentos que guíen dicha observación y posibiliten conclusiones respaldadas al respecto.

9. La acreditación de bloques de competencias incrementa la motivación de la persona candidata acerca del proceso de reconocimiento y de formación al permitir establecer itinerarios con mayor nivel de profesionalización.

10. La acreditación de módulos transversales responde a una valoración de la persona candidata como profesional más allá de su competencia técnica y ajustada a su competencia como trabajador (organización empresa, papel en la organización, búsqueda de empleo, tipo de contratación, instituciones, etc.), competencias laborales de las que carecen los jóvenes y que llevan a cabo en la formación inicial.

\section{Referencias Bibliográficas}

Arias, I. (2007). "El sistema de acreditación en el País Vasco. Reconocimiento de la competencia en el País Vasco". En Congreso Internacional sobre Reconocimiento y Acreditación de las Competencias (CREAC). Oviedo: Cámara de Comercio de Asturias.

Arraiz, A. y Sabirón, F. (2007). "El portafolio etnográfico: una herramienta facilitadota del aprendizaje a lo largo de la vida". Revista española de orientación y psicopedagogía, 18, 1, 65-72.

BOA (2004). Resolución del Viceconsejero de Formación Profesional y aprendizaje permanente por la que se establecen las condiciones, metodologías y procedimientos para la realización de pruebas para la obtención de los títulos de Técnico y Técnico Superior a través del dispositivo de 
evaluación y reconocimiento de la competencia. Zaragoza: Boletín Oficial de Aragón 29 de noviembre de 2004.

BOA (2006). Orden de 19 de diciembre de 2006, de los Departamentos de Economía, Hacienda y Empleo y de Educación, Cultura y Deporte, por la que se establece el procedimiento de evaluación y acreditación de competencias adquiridas por la experiencia laboral o el aprendizaje no formal. Zaragoza: Boletín Oficial de Aragón, de 15 de enero de 2007.

BOA (2007). Resolución del Viceconsejero de Formación Profesional y aprendizaje permanente por la que se establecen las condiciones, metodologías y procedimientos para la realización de pruebas para la obtención de los títulos de Técnico y Técnico Superior a través del dispositivo de evaluación y reconocimiento de la competencia. Zaragoza: Boletín Oficial de Aragón 26 de noviembre de 2007.

BOA (2007). Orden de 29 de octubre de 2007, de los Departamentos de Economía, Hacienda y Empleo y de Educación, Cultura y Deporte, por la que se establece, con carácter experimental el Procedimiento de Evaluación y Acreditación de Competencias adquiridas por la experiencia laboral o el aprendizaje no formal. Aragón: Boletín Oficial de Aragón, de 26 de noviembre de 2007.

BOCA (2007). Orden de 23 de abril de 2007, por la que se establece para el año 2007, con carácter experimental y limitado, el procedimiento de reconocimiento, evaluación y acreditación de competencias del Título de Técnico en Cocina, adquiridas por la experiencia laboral o el aprendizaje no formal. Santa Cruz de Tenerife: Boletín Oficial de Canarias, de 5 de Junio de 2007.

BOE (2002). Ley Orgánica 5/2002, de 19 de junio, de Cualificaciones y Formación Profesional. Madrid: Boletín Oficial del Estado, de 20 de junio de 2002.

BOE (2002). Real Decreto 1326/2002, de 13 de diciembre, por el que se modifica Real Decreto 375/1999, de 5 de marzo, por el que se crea el Instituto nacional de las Cualificaciones. Madrid: Boletín Oficial del Estado, de 14 de diciembre de 2002.

BOE (2003). Real Decreto 942/2003, de 18 de junio por el que se regulan las condiciones básicas que deben reunir las Pruebas para la obtención de títulos de Técnico y Técnico Superior de Formación Profesional Específica. Madrid: Boletín Oficial del Estado, de 31 de julio de 2003.

BOE (2003). Real Decreto 1046/2003, de 1 de agosto, por el que se regula el subsistema de formación profesional continua. Madrid: Boletín Oficial del Estado, de 12 de septiembre de 2003.

BOE (2006). Ley Orgánica 2/2006, de 3 de mayo, de Educación. Madrid: Boletín Oficial de Estado, de 4 de mayo de 2006.

BOE (2007). Real Decreto 395/2007, de 23 de marzo, por el que se regula el subsistema de formación profesional para el empleo. Madrid: Boletín Oficial del Estado, de 11 de abril de 2007.

BOPV (2004). Decreto 70/2004, de 27 de abril, por el que se regulan las pruebas para la obtención de los Títulos de Técnico y Técnico Superior de Formación Profesional Específica. Vitoria: Boletín Oficial del País Vasco, de 5 de mayo de 2004.

Consejería de Educación (2006). Informe sobre el desarrollo del procedimiento de Evaluación y acreditación de competencias adquiridas por la experiencia laboral, convocado por orden de 19 de Diciembre de 2006. Documento Interno Agencia de la Certificación Profesional de la Competencia de Aragón.

Departament d' Educació (2007). Informe del proceso de acreditación de las competencias profesionales. 2007. Documento Interno Instituto Catalán de las Cualificaciones Profesionales. (Documento policopiado).

Departamento de Educación Universidades E Investigación (2004). Agencia Vasca para la Evaluación de la Competencia y la Calidad de la Formación Profesional. Dispositivo de Reconocimiento de la 
Competencia del País Vasco. Vitoria. http://www.hezkuntza.ejgv.euskadi.net/r43-2333/es/ 9 de diciembre de 2007.

Departamento de Educación, Universidades e Investigación (2004). Plan Vasco de Formación Profesional. Vitoria. Página web: http://www.hezkuntza.ejgv.euskadi.net/r43-2627/es/contenidos/informacion/plan_vasco_fp/es_2018/adjuntos/plan2004_1_c.pdf Consulta: 9 de diciembre de 2007.

Departamento de Educación, Universidades e Investigación (2004). Dispositivo de reconocimiento de la competencia. Modelo y principios generales. Vitoria: Agencia Vasca, (Documento policopiado).

DOGC (2004). Resolució ENS/690/2004, de 16 de març, per la qual es convoca la realització de les proves per a l'obtenció dels títols de tècnic i tècnic superior de determinats cicles formatius de formació professional especifica. Barcelona: Diario Oficial de Generalitat de Catalunya, de 25 de marxo de 2004.

Grupo técnico de trabajo del proyecto ERA (2004). El proyecto ERA y la validación de los aprendizajes no formales e informales. Revista Diálogos. Certificación y validación de aprendizajes y competencias, $\mathrm{n}^{\circ}$ 39-40. Volumen 3.

Ibarra, M. y Sánchez Rojas, C. (1999). Posibilidades del portafolios en Auto-orientación Profesional. Revista española de orientación y psicopedagogía, 10, 17, 63-82.

MEC (2004). Proyecto ERA. Evaluación, reconocimiento y acreditación de las competencias profesionales. Memoria final del proyecto ERA. Disponible en http://www.mec.es/educa/incual/ice_recAcr.html (7 mayo 2007). http://www.hezkuntza.ejgv.euskadi.net/r432359/es/contenidos/ informacion/agencia/es_1967/adjuntos/resolu.pdf.

MEC (2003). Guía del candidato, guía del orientador y guía del evaluador Disponible en http://www. mec.es/educa/incual/pdf/rec/10_anexo4guias.pdf (5 mayo 2007).

Moreno, F. J. (2007). Reconocimiento, evaluación y acreditación de la competencia profesional de los trabajadores forestales de Castilla-La Mancha. En Congreso Internacional sobre reconocimiento $y$ acreditación de las competencias (CREAC). Oviedo: Cámara de Comercio de Asturias.

Ranchal, F. (2007). Acreditación de experiencias adquiridas por la experiencia profesional. En Congreso Internacional sobre Reconocimiento y Acreditación de las Competencias (CREAC). Oviedo: Cámara de Comercio de Asturias.

Servicio Público de Empleo de Castilla-La Mancha (SEPECAM) (2007). Proyecto de reconocimiento, evaluación y acreditación de la competencia profesional de los trabajadores forestales de Castilla-La Mancha. (Documento).

Fecha de recepción: 30-01-2008

Fecha de revisión: 16-07-2009

Fecha de aceptación: 21-10-2009 